\title{
Experiments on AC Electrokinetic Pumping of Liquids Using Arrays of Microelectrodes
}

\author{
P. García-Sánchez, A. Ramos \\ Departamento de Electrónica y Electromagnetismo. \\ Universidad de Sevilla. 41012 Sevilla, Spain \\ N. G. Green and H. Morgan \\ School of Electronics and Computer Sciences. \\ University of Southampton. SO17 1BJ, UK
}

\begin{abstract}
Net fluid flow induced by ac potentials applied to arrays of co-planar interdigitated microelectrodes is reported. Two types of microelectrode structures have been employed: arrays of unequal width electrodes subjected to a single ac signal, and arrays of identical electrodes subjected to a traveling-wave potential. A square glass chamber was constructed around the electrode arrays and filled with a concentration of $\mathrm{KCl}$ in water of conductivity around $1 \mathrm{mS} / \mathrm{m}$. A map of the fluid velocity as a function of voltage (0-8 Vpp) and frequency $(0.1-100 \mathrm{kHz})$ is presented for the traveling-wave array. In both microstructures, two fluid flow regimes have been observed: at small voltage amplitudes the fluid moves in a certain direction, and at higher voltage amplitudes the fluid flow is reversed. The fluid flow seems to be driven at the level of the electrodes in the two flow regimes. The observations at low voltages are in qualitative accordance with an ac electroosmotic model based upon the Debye-Hückel theory for the double layer.
\end{abstract}

Index Terms — Micropumps, electrohydrodynamics, liquids.

\section{INTRODUCTION}

THE development of 'lab-on-a-chip' devices or micro total analysis systems ( $\mu \mathrm{TAS})$ demands the precise control of fluids in small channels (a process called microfluidics) [1]. Many techniques have been developed to pump liquids in microsystems, including micromechanical methods [2], electroosmosis [3], electro-wetting [4], thermocapillary pumping [5], electrohydrodynamic pumping [6] and ac electroosmosis [7]. In the latter, electrolytic solutions are pumped using arrays of micro-electrodes subjected to a low potential ac signal [8-12]. The liquid is driven at the surface of the electrodes, where the electric field produces a lateral force on the induced charge in the double layer, moving the fluid [13-18].

In this paper, two different electrode arrays have been used to generate fluid flow, using either a traveling electric field or a single phase ac potential with an asymmetric electrode array. A periodic array of electrodes is fabricated onto an insulating substrate (glass) as shown in Figure 1. Figure 1a shows the asymmetric electrode array which is energized with a single phase potential. In Figure $1 \mathrm{~b}$ the electrodes are of equal width and gap and energized with a 4-phase ac signal. Above the electrode array there is an electrolyte (such as $\mathrm{KCl}$ ), with conductivity $\sigma$ and permittivity $\varepsilon$. At the interfaces between the

Manuscript received on 19 September 2005, in final form 17 April 2006. metal and electrolyte, and glass and electrolyte, double layers are formed. The characteristic thickness of a double layer is given by the Debye length, $\lambda_{D}$, and in most cases it is negligibly small $(\sim 10 \mathrm{~nm})$ compared to the other lengths in the system. When an ac voltage is applied to the electrodes, an electrical current is established in the solution which leads to the formation of a transient excess charge in the electrical double layer. This is shown schematically in Figure 2 for the asymmetric electrode array. If the ac electric field is nonuniform, the component of the electric field normal to the electrode surface induces the charge in the double layer at the electrode/electrolyte interface, whilst the tangential component produces a body force on the induced charge, thus moving the fluid in two rolls over the electrodes $[9,16]$. The generated velocity field has a steady-state component which is observed experimentally. In a symmetric electrode array (e.g. two parallel plates) the global average fluid motion is zero. To achieve unidirectional fluid movement the electrodes array is made non-symmetric as shown in Figure 1a [7]. This asymmetry leads to fluid rolls of different sizes over each electrode, leading to net fluid flow as shown schematically in Figure $2 b$.

Figure 3 shows the case of the traveling electric field, where the electrodes are symmetric, and driven by a traveling ac voltage. The electric field generates an induced charge in the double layer, as for the asymmetric array. Due to the finite 

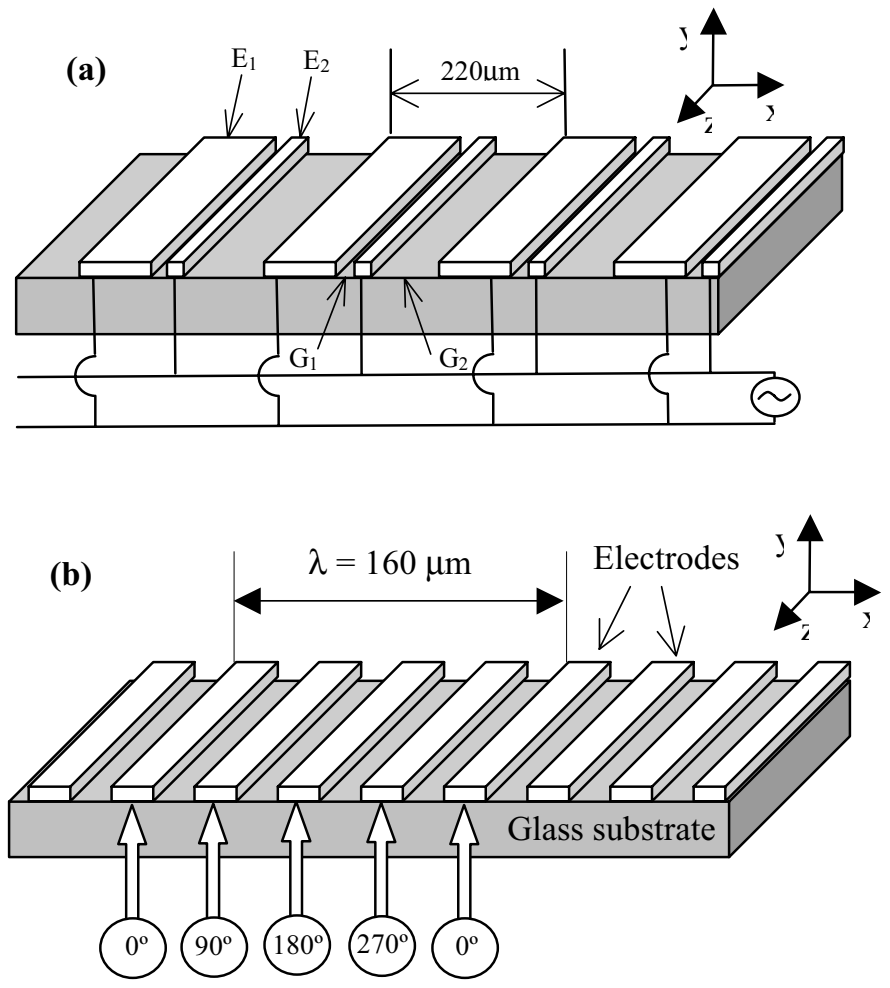

Figure 1. Schematic diagrams of: (a) asymmetric electrode array. (b) Traveling-wave electrode array.

time during which the double layer is charged, a delay exists between the time of maximum induced charge and the maximum applied signal. The electric field acts on the charge pulling the fluid in the direction of the traveling wave, giving rise to net fluid flow $[11,12]$.

In this paper, we present experimental observations of fluid flow in both types of micro-electrode arrays. We have paid particular attention to the traveling-wave array, where we have extended previous experimental results [12] to a greater range of voltages. In addition we have explored the frequency dependence of the fluid flow.

\section{THEORY AT LOW VOLTAGE}

To a first approximation we assume that the applied voltage is low enough such that Faradaic currents from electrode to electrolyte are absent. We also assume that the frequency of the applied signal is low enough, i.e. $\omega \lambda_{D}{ }^{2} / D<<1$, so that the double layer is in quasi-equilibrium. Here $D$ is the mean diffusion coefficient of the ions and $\lambda_{D}^{2} / D=\varepsilon / \sigma$ is the time an ion takes to travel the Debye length by diffusion. For periods of the applied signal much greater than $\lambda_{D}{ }^{2} / D$, the ions can equilibrate locally. Typical relaxation times for our saline solutions are between $0.7 \mu \mathrm{s}$ and $0.07 \mu \mathrm{s}$, for conductivities between $1-10 \mathrm{mS} / \mathrm{m}$. The periods of the applied signals of the experimental section are much greater, 0.1-10 ms. Under these conditions the bulk electrolyte behaves in a resistive manner and the double layer can be considered to behave as an ideal
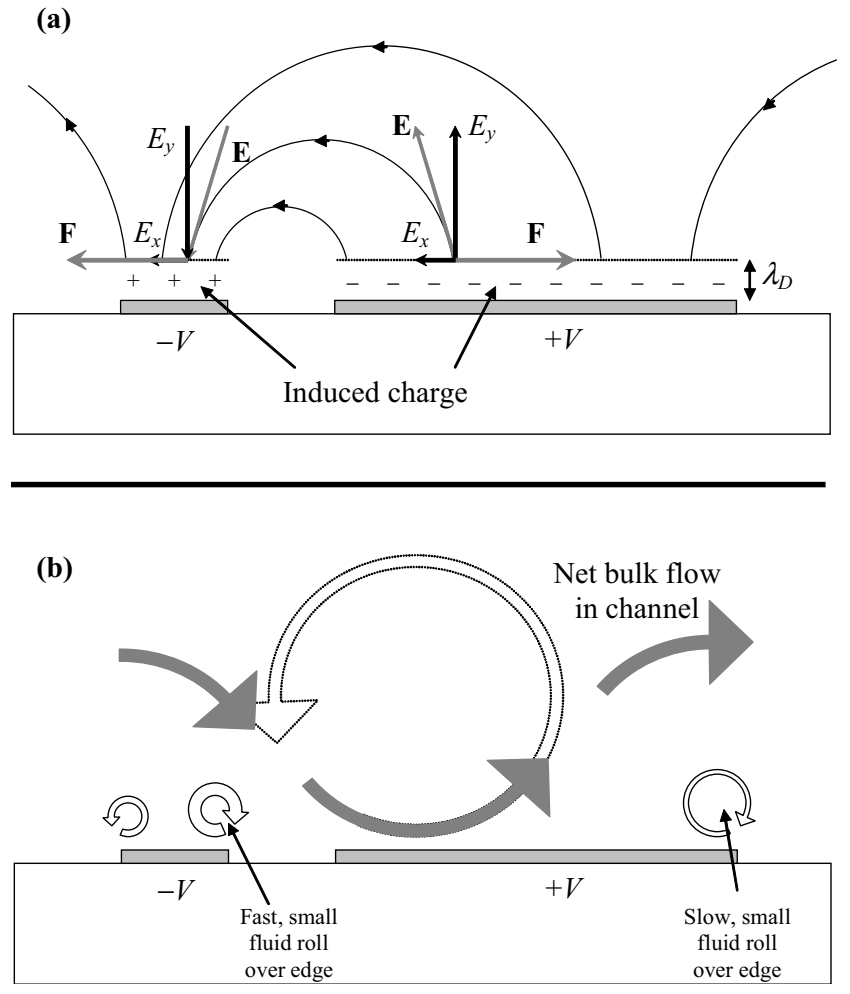

Figure 2. Schematic diagram of the physical mechanism of ac electroosmosis in an asymmetric electrode array

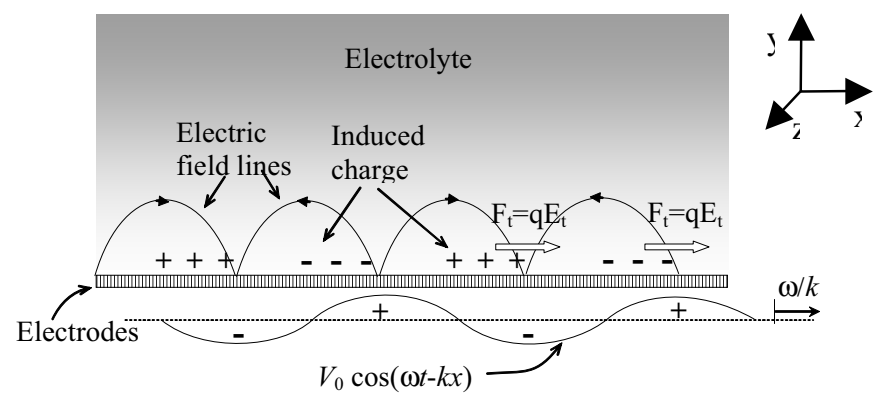

Traveling wave applied potential

Figure 3. Schematic diagram illustrating the physical mechanism of traveling-wave electroosmosis

capacitor. Therefore, the electrical potential $\phi$ in the bulk electrolyte satisfies Laplace's equation

$$
\nabla^{2} \phi=0
$$

The boundary condition on the electrode surface describes the charging of the double layer due to the current in the bulk. For sufficiently low voltage there is a linear relationship between the surface charge and the voltage drop across the double layer. In this case, the surface charge conservation equation can be written using phasors as [16]

$$
\sigma \frac{\partial \phi}{\partial y}=i \omega C_{D L}\left(\phi-V_{j}\right)
$$

where $C_{D L}$ is the capacitance per unit of area of the total double layer (diffuse plus compact or Stern layers), $i$ is the 
imaginary unit, $\phi$ is the potential just outside the double layer, and $V_{j}$ is the potential applied to electrode $j$. At the interface between the electrolyte and the glass a similar boundary condition holds, however, in this case the boundary condition can be simplified to $\partial \phi / \partial y=0$, i.e. no current flows to the glass/electrolyte interface [16]. The boundary condition at $y \rightarrow \infty$ is that the potential tends to zero.

Owing to electrode polarization, the electric field in the bulk electrolyte is frequency-dependent. When the frequency is low, most of the applied voltage is dropped across the double layer (across the capacitor). Conversely, when the frequency is high, most of the applied voltage is dropped across the bulk electrolyte. The typical transition frequency can be estimated from simple circuit theory to be $\omega_{0} \sim \sigma \lambda_{D} / \varepsilon l$ (with $l$ a characteristic system length), which is several orders of magnitude smaller than the charge relaxation frequency $\sigma / \mathcal{E}$. For typical values in microsystems, $f_{0}=\omega_{0} / 2 \pi \square 10^{3} \mathrm{~Hz}$ when $l=10 \mu \mathrm{m}, \quad \sigma=10^{-3} \mathrm{~S} / \mathrm{m}, \varepsilon=80 \varepsilon_{0}$ Fluid flow due to ac electro-osmosis is observed in the region of this characteristic frequency $\omega_{0}[8,13,17]$.

Once the electric potential has been solved, the electroosmotic fluid velocity at the surface of the electrodes can be calculated. For diffuse double layers in quasi-equilibrium on perfectly polarisable metal surfaces, the electro-osmotic slip velocity is given by the Helmholtz-Smoluchowski formula [19]

$$
u=\frac{\varepsilon \Delta \phi}{\eta} E_{x}
$$

where $\eta$ is the viscosity of the fluid, $\Delta \phi$ is the potential drop across the diffuse double layer and $E_{x}$ is the tangential electric field outside the double layer. For our problem, both $\Delta \phi$ and $E_{x}$ are oscillating functions of time, of frequency $\omega$. The timeaveraged horizontal fluid velocity at the interface between the double layer and the bulk is $[15,16]$

$$
\langle u\rangle=-\frac{\varepsilon}{4 \eta} \Lambda \frac{\partial\left|\Delta \phi_{D L}\right|^{2}}{\partial x}
$$

where $\Delta \phi_{\mathrm{DL}}$ is the voltage drop across the total double layer, $\Lambda$ is given by $\Lambda=C_{s} /\left(C_{s}+C_{d}\right)$ and $C_{d}$ and $C_{s}$ are the capacitances of the diffuse and Stern or compact layers, respectively. For the glass/electrolyte interface, an estimate of the potential drop across the diffuse double layer shows that this is negligibly small and, from equation (3), the electroosmotic velocity on the glass is negligible.

To obtain the velocity in the bulk, the Navier-Stokes equation must be solved. For micro-systems the Reynolds number is usually very small, so that the convection term in the equations can be neglected. For the time-averaged velocity, and in the absence of externally applied body forces, the equations reduce to:

$$
\eta \nabla^{2} \mathbf{u}-\nabla p=0, \quad \nabla \cdot \mathbf{u}=0
$$

where $p$ is the pressure and $\mathbf{u}$ the velocity field. The boundary conditions for these equations at $y=0$ are: (a) the tangential velocity is equal to the slip velocity on the electrodes, given by eq. (4); (b) the tangential velocity is zero at the glass; (c) the normal velocity is zero for any $x$ at $y=0$ (electrodes and glass). A thorough analysis of the linear theory for the traveling wave and asymmetric pumps can be found in other references $[9,11,12]$.

\section{EXPERIMENTAL DETAILS}

The asymmetric array use for pumping is shown in Figure 1a employed. This array consists of ten pairs of electrodes $\left(E_{1}\right.$ and $\left.E_{2}\right)$ of width 100 and $10 \mu \mathrm{m}$ separated by $10 \mu \mathrm{m}$ gap $\left(G_{l}\right)$. The distance between pairs of electrodes is $100 \mu \mathrm{m}\left(G_{2}\right)$, so the spatial periodicity is $220 \mu \mathrm{m}$. The array has a total length of $2100 \mu \mathrm{m}$. The electrodes are made from a sandwich of titanium gold titanium, 10/100/10 nm thick. Each pair of asymmetric electrodes is subjected to an ac potential difference.

The microelectrode array used in the traveling-wave (TW) experiment is shown in Figure 1b. The device consists of an array 20 interdigitated electrodes fabricated on a planar glass slide using photolithography. The electrodes are $20 \mu \mathrm{m}$ wide separated by $20 \mu \mathrm{m}$ gaps. Each electrode is driven by an ac voltage of amplitude $V_{0}$ and frequency $\omega$. The voltage on consecutive electrodes is phase shifted by $90^{\circ}$, as shown in Figure 1. This produces a traveling wave potential with a wavelength of $160 \mu \mathrm{m}$.

A square glass chamber was constructed around the electrode arrays and filled with a solution of $\mathrm{KCl}$ in water of conductivity $1.3 \mathrm{mS} / \mathrm{m}$. For the traveling wave device the chamber height was $200 \mu \mathrm{m}$, whilst the asymmetric device had a chamber height of $260 \mu \mathrm{m}$.

Fluid flow was observed both from above and from the side (along the length of the electrodes). A microscope objective and camera were pointed horizontally along the electrodes, so that the electrodes could be imaged in cross section. Fluorescent latex particles (500 $\mathrm{nm}$ diameter) were suspended in the electrolyte and used as flow tracers.

Observations of the fluid flow were recorded on video and transferred to a computer. The fluid velocity measurements were carried out by particle tracking velocimetry (PTV) written in Matlab.

\section{RESULTS}

When an ac voltage is applied to the electrode arrays, fluid flow is driven at the electrode surface in both cases. An example of fluid flow above the electrodes for the traveling wave pump is shown in Figure 4. This image is of fluid motion viewed from the side with the electrodes in the bottom of the image. The fluorescent tracer particles are used to plot the stream-lines by superimposing successive video frames (50 in this case). The chamber is closed at both ends so that the fluid recirculates. This can be seen in the figure where the tracer particles at the top move in the opposite direction to those above the electrodes in the bottom half of the chamber. 


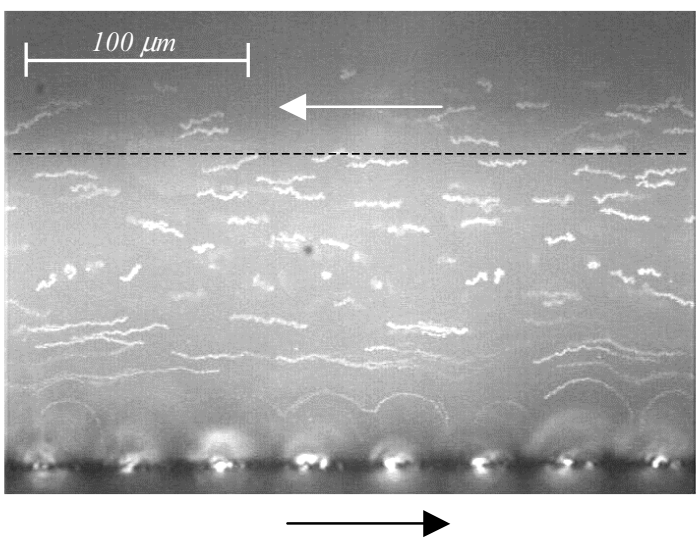

Figure 4. Particle paths for the traveling-wave array for an applied signal of $10 \mathrm{kHz}$ and $5 \mathrm{Vpp}$. The black arrow indicates the direction of the fluid flow at the electrode level, and the white arrow the direction at the top of the chamber. A dashed line is placed at the height where the fluid velocity in Figure 6 is measured.

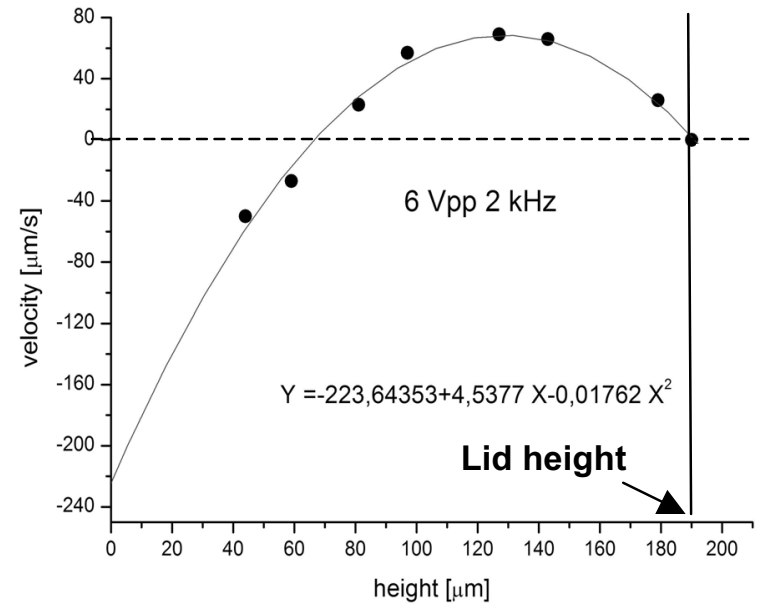

Figure 5. Horizontal velocity versus height for an applied signal of $2 \mathrm{kHz}$ and $6 \mathrm{~V}_{\mathrm{pp}}$ (traveling wave array). The flow profile is similar to a Couette-Poiseuille flow (solid line).

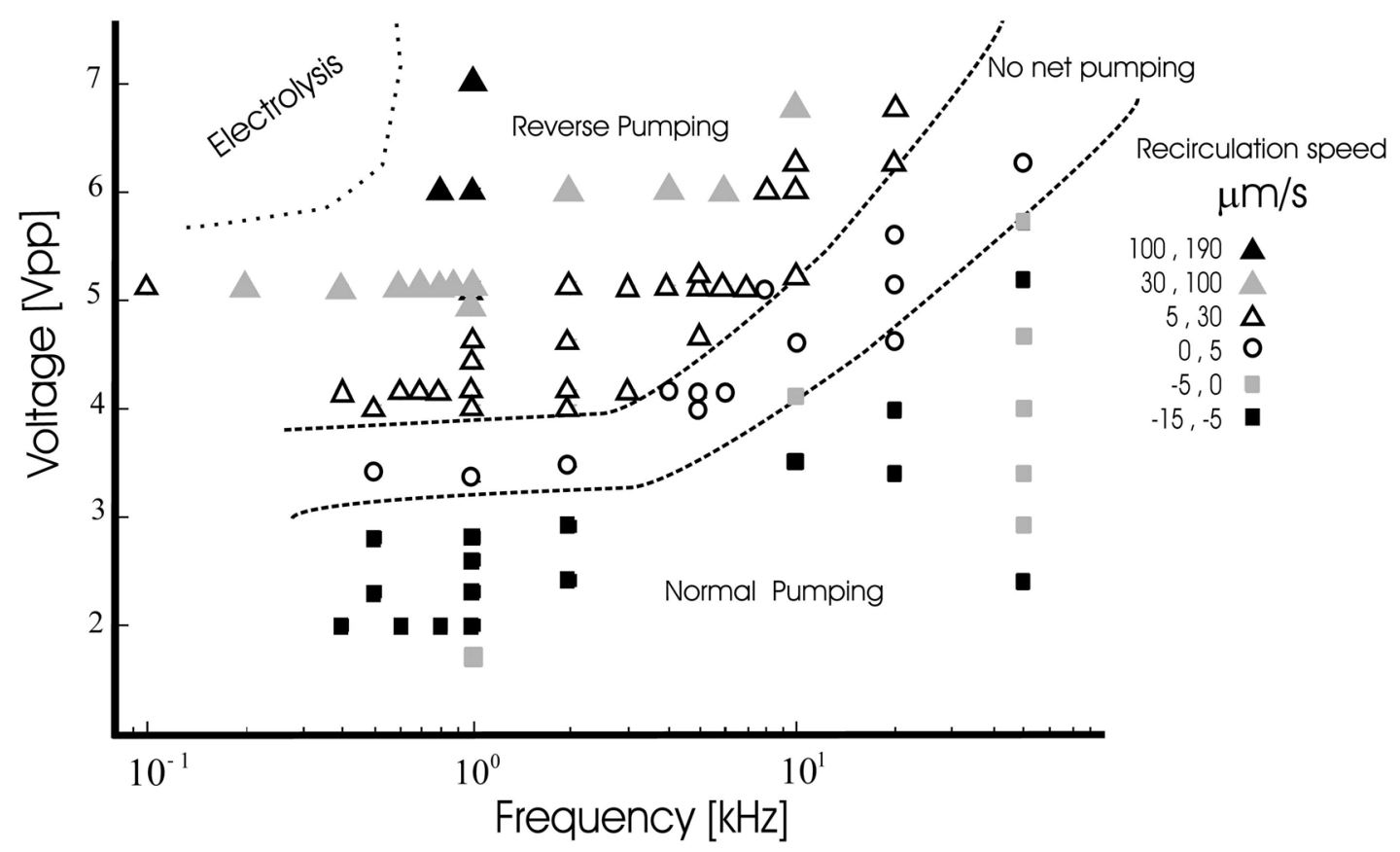

Figure 6. Velocity map for the traveling-wave array of microelectrodes in the plane voltage versus frequency. Velocities measured at a height of $140 \mu \mathrm{m}$.

\subsection{TRAVELING WAVE ARRAY}

A typical plot of the horizontal fluid velocity measured as a function of height above the electrodes is shown in Figure 5. This was taken for a voltage of $6 \mathrm{~V}$ (peak to peak) at a frequency of $2 \mathrm{kHz}$. Similar profiles were obtained for other frequencies and voltages. The experimental velocity profile can be fitted to a parabola with the maximum speed at the electrodes (solid line in the figure). This velocity profile has zero net flux, as expected for a $2 \mathrm{D}$ flow when the chamber is closed at both ends (right and left ends in Figure 4). This profile corresponds to the theoretical prediction obtained from the Stokes equation with no volumetric force and non-slip velocity condition at the top boundary and slip condition at the bottom boundary. The experimental curve shows that the fluid is driven at the electrodes, and recirculates at the top of the chamber with a total flux equal to zero. The average velocity at the level of the electrodes can be estimated by extrapolating the velocity profile as shown in the figure.

The pump behavior was characterized as a function of voltage and frequency by measuring the fluid velocity at a height of $140 \mu \mathrm{m}$ above the electrodes. At this height the fluid 


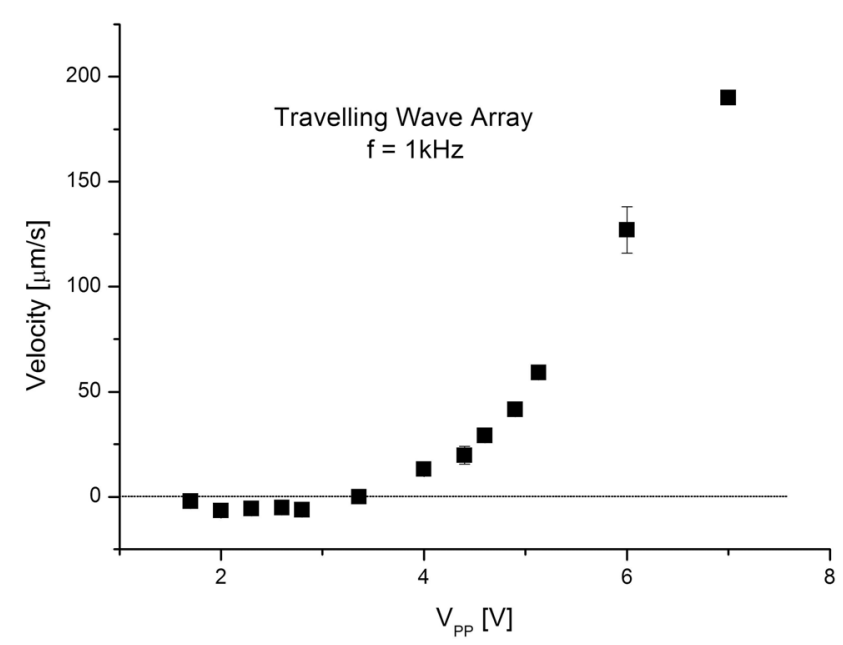

Figure 7. Horizontal velocity at a height of $140 \mu \mathrm{m}$ versus voltage, for a given frequency of $1 \mathrm{kHz}$ (traveling-wave array)

is moving in the opposite direction to that at the electrode surface. Measurements at this height were considered to be representative of the global behavior of the pump as indicated by the profile of Figure 5 .

A 2-D map of these measurements plotted in the voltage versus frequency domain is shown in Figure 6. Values of velocity are negative when the fluid is dragged on the electrodes in the direction of the traveling electric field. At low voltages, the fluid is dragged in this direction, i.e. negative velocity values. We refer to this mode of behavior as Normal Pumping. This is the mode of pumping that is predicted by the low voltage theory of ac electroosmosis [11,12]. At higher voltages, the fluid is driven in the opposite direction, a mode of behavior we designate as Reverse Pumping. The physical mechanism responsible for this behavior is not clear at present.

In Figure 6 four separate regions can be distinguished, corresponding to: normal pumping, reverse pumping, no net pumping and electrolysis. The latter corresponds to a region of very low frequencies and high voltages which is generally destructive owing to bubble formation because of electrolysis. There is a region of voltage where the pumping mechanism changes from normal to reverse pumping. No net flow was observed in this region. Fluid rolls occurred over the electrodes but these did not lead to unidirectional fluid flow. Therefore, we designate this region as No Net Pumping.

Figure 7 shows the recirculation magnitude of the velocity for the pump at a frequency of $1 \mathrm{kHz}$ as a function of voltage. At low voltages normal pumping is observed. The velocity amplitude increases only slightly with voltage until saturation and then no net pumping is observed. At a voltage of $4 \mathrm{~V}$ peak to peak, the fluid velocity begins to increase again (in the opposite direction) as reverse pumping occurs. The velocity amplitude then increases rapidly with voltage as shown in the figure. At $7 \mathrm{~V}$ peak to peak the fluid speed is $200 \mu \mathrm{m} / \mathrm{s}$, corresponding to an average velocity of $600 \mu \mathrm{m} / \mathrm{s}$ at the level of the electrodes.

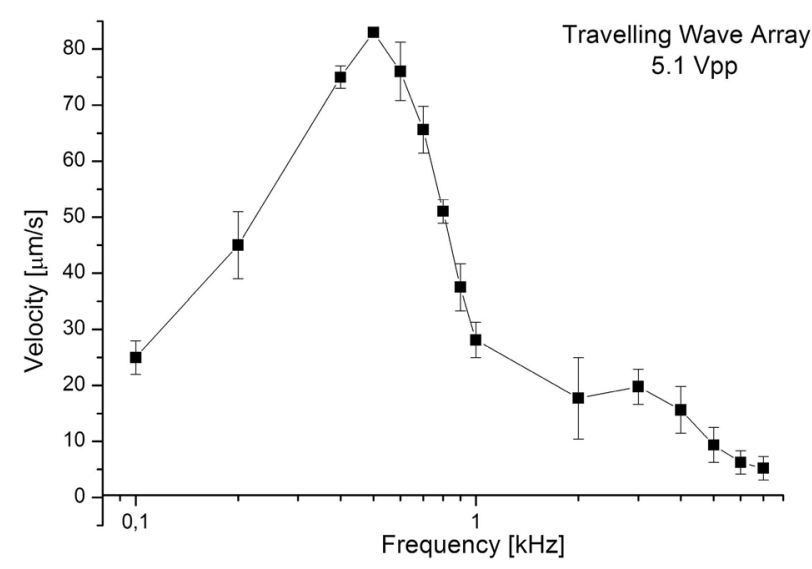

Figure 8. Horizontal velocity at a height of $140 \mu \mathrm{m}$ versus frequency, for a given voltage of $5.1 \mathrm{~V}_{\mathrm{pp}}$ (traveling-wave array)

A plot of the velocity against frequency at a constant voltage $\left(5 \mathrm{~V}_{\mathrm{pp}}\right)$ is shown in Figure 8 . The velocity reaches a maximum at a frequency of approximately $500 \mathrm{~Hz}$.

In previous work, we developed a linear theory for traveling wave electroosmotic pumping for devices energized with a 4phase ac signal [12]. Applying this theory to this particular device predicts that the maximum pumping velocity at the electrodes is $U=345 \Lambda V_{p p}^{2} \mu \mathrm{m} / \mathrm{s}$, where the parameter $\Lambda$ is the ratio of the voltage across the diffuse layer compared with the total voltage across the double layer. The fluid velocity at the level of the electrode was determined by extrapolation of the experimental data. For the electrode array used in this work, and at a voltage of $V_{p p}=2$ volts and $f=600 \mathrm{~Hz}$ the measured velocity was $38 \mu \mathrm{m} / \mathrm{s}$. Comparison with theory shows that $\Lambda \approx 0.03$. This small value for $\Lambda$ can be in part explained by the fact that the oxide layer which forms on top of the titanium electrode reduces the voltage across the diffuse layer.

In [12] we reported results using this electrode array but with a different electrolyte conductivity of $2 \mathrm{mS} / \mathrm{m}$. The maximum velocity for the low voltage region was observed at a frequency in the range of $1-2 \mathrm{kHz}$. In the present work, (electrolyte conductivity is $1.3 \mathrm{mS} / \mathrm{m}$ ), the maximum velocities within the low voltage region occurred at a lower frequency of around 500-600 Hz. This trend is in accordance with the theoretical prediction given in [12] for the dependence of the frequency of maximum velocity with conductivity. The linear model predicts that the maximum fluid velocity increase with conductivity according to $\omega=1.45 \sigma k_{0} / C_{D L}$, with $k_{0}=2 \pi / L$, where $L=$ characteristic wavelength of the traveling wave device $(160 \mu \mathrm{m})$.

\subsection{ASYMMETRIC ARRAY}

Pumping of electrolytes using an asymmetric electrode array has been extensively characterized by other groups [7,10,20]. In this work, we plot data from an asymmetric electrode pump 


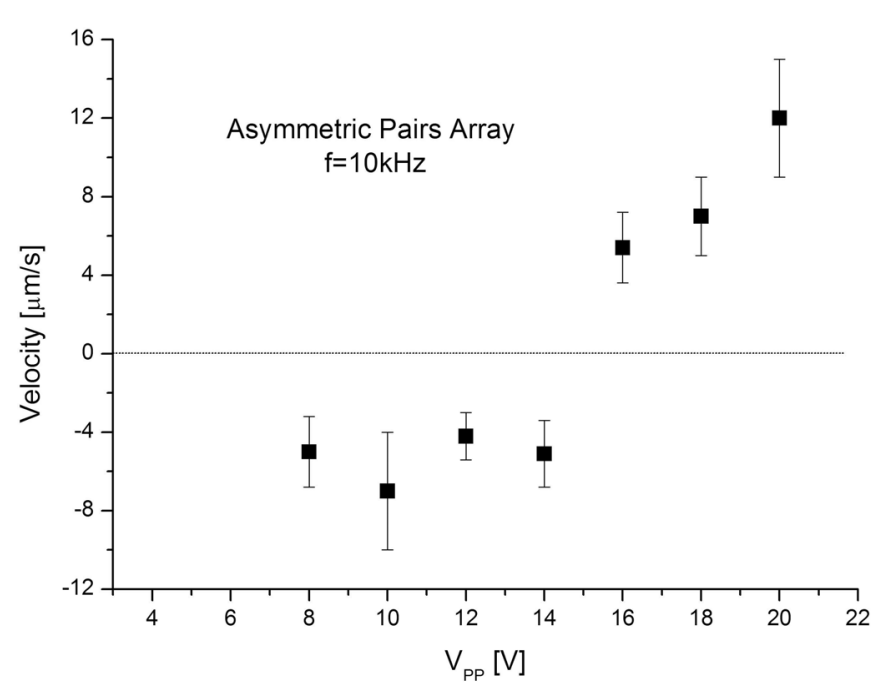

Figure 9. Horizontal velocity at a height of $210 \mu \mathrm{m}$ as a function of voltage (asymmetric array).
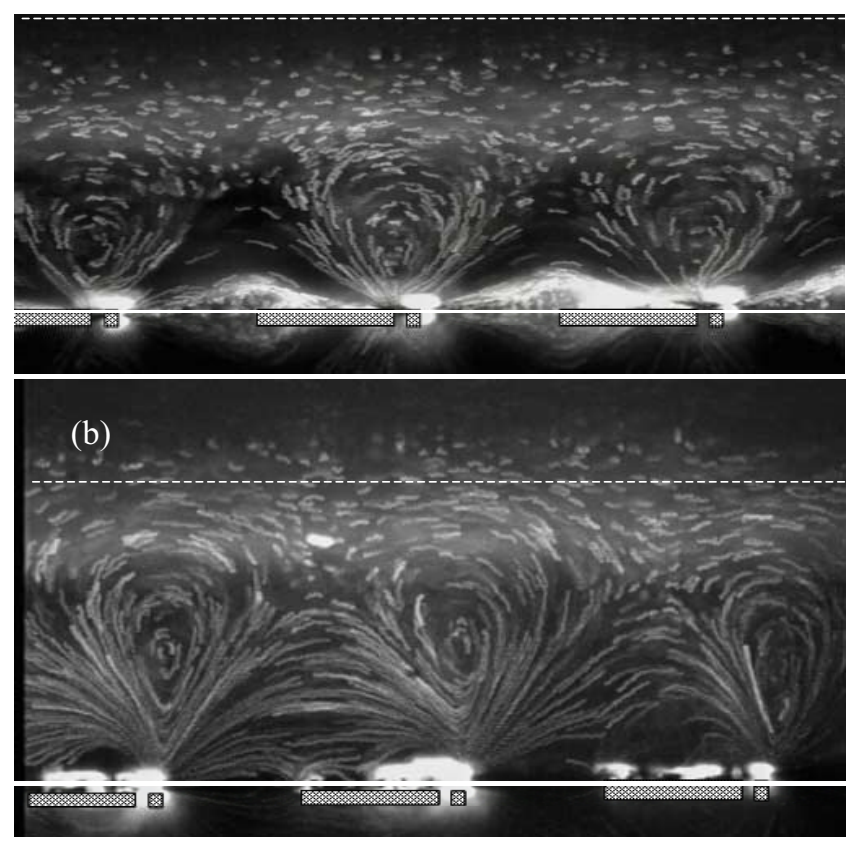

Figure 10. Particle paths for the asymmetric array. (a): $10 \mathrm{kHz}$ and 8 $\mathrm{V}_{\mathrm{pp}}$. (b): $10 \mathrm{kHz}$ and $18 \mathrm{~V}_{\mathrm{pp}}$. The electrodes are drawn in the images. Dashed lines are placed at the height where the fluid velocity was measured.

for comparison with the traveling wave array using electrodes made from the same metal and with the same electrolyte conductivity. Figure 9 is a plot of the magnitude of the fluid velocity as a function of voltage, measured at a height of 210 $\mu \mathrm{m}$ above the electrodes at a frequency of $10 \mathrm{kHz}$. The velocity at this height is considered representative of the global behavior of the pump since it is in the region above the fluid rolls. At the frequency of $10 \mathrm{kHz}$ the fluid flow was observed to be near the maximum for this design of electrode array. Similar to Figure 7, the electrolyte moves across the electrodes in one direction at low voltages and changes direction at a transition voltage of approximately $15 \mathrm{~V} \mathrm{pk}$ to pk. At low voltages the net fluid flow direction corresponds to that predicted by linear theory, i.e. from small to large electrode as shown in Figure 2b. This is termed normal pumping. At higher voltages the fluid reverses direction, as for the traveling wave pump.

Figures $10 \mathrm{a}$ and $10 \mathrm{~b}$ show the experimental fluid streamlines for normal pumping and reverse pumping in each case. The large fluid roll that extends into the bulk in each case has a typical size around $220 \mu \mathrm{m}$, that is the spatial periodicity of the array. When the pumping changes to reverse mode, the rolls over the electrodes change, both in shape and position on the electrodes. The rolls in the reversal mode seems to be slightly shifted to the right.

\section{CONCLUDING REMARKS}

The traveling-wave electrode array is generally used to move particles using traveling wave dielectrophoresis, TWD [21]. We can compare particle motion due to fluid flow and that due to TWD motion. Using the theoretical results given in [22], the horizontal traveling wave dielectrophoretic velocity at a height $y \geq 1.5 d$ (in water) is given by

$$
v_{T W D}=4.35 \times 10^{-8} \operatorname{Im}(\tilde{f}) \frac{a^{2} V_{p p}^{2}}{d^{3}} e^{-(\pi / 2 d) y} \mathrm{~m} / \mathrm{s}
$$

where, $a$ is the particle radius and $d$ the electrode width (in meters), and $\operatorname{Im}(\tilde{f})$ the imaginary part of the ClausiusMossotti factor. This equation shows that at large distances from the electrode $(y>>d)$ the electric field is close to zero. Therefore at the point of observation $(140 \mu \mathrm{m}$ above the electrodes) the TWD (and DEP) velocities are negligible (smaller than Brownian motion). Therefore the fluid velocity measurements are not affected by dielectrophoresis at this height. Even at heights around $1.5 d$ the traveling wave dielectrophoretic motion is negligible. For particles 10 times larger, at $6 \mathrm{~V}_{\mathrm{pp}}$ and at a height around $d$ the dielectrophoretic velocity is less than $30 \mu \mathrm{m} / \mathrm{s}$. Generally traveling wave DEP is observed when particles undergo negative DEP and have a non-zero imaginary component of the dipole. Practically, this occurs at frequencies much greater than $1 \mathrm{kHz}$. At high frequencies the ac electroosmotic fluid velocities are much lower, so that TWD dominates.

Comparison of the asymmetric pump and the traveling wave pump is not simple because, although they both are driven by ac electroosmosis, the electric field and geometry are different in each case. However, some general observations can be made. The fluid velocity is much greater for the traveling wave device than for the asymmetric electrode (for similar voltages applied to each electrode). The experimental streamlines can also be compared. The fluid flow for the traveling-wave device is more uniform, and this can be an advantage in circular chromatographic applications. In addition, both pumps require low-conductivity electrolytes to function and also operate optimally at low frequencies with bare electrodes. It has been shown that if the electrodes are 
covered by an extremely thin layer of insulator, the characteristic frequency of the device is higher [11]. This characteristic frequency is given by $\omega=\sigma / \varepsilon$, if there is a thick dielectric between electrode and electrolyte [24].

The current theory of ac electroosmosis is based on a linear approximation to the double electrical layer, an assumption which is only valid for low applied voltages. The parameter $\Lambda$ which determines the value of the potential dropped across the diffuse part of the double layer was found to be smaller than in other experiments [16]. This discrepancy requires further analysis and is probably related to the non-linear behavior of the double layer and the oxide which grows on the Ti electrodes. The incorporation of a more complete model of the electrode/electrolyte interface is required for a comprehensive analysis of the experimental data

Concerning to the dependence of fluid velocity with the dimensions of the array, the linear theory predicts that the slip velocity on the electrodes increases inversely proportional to the length of the unit cell of the array. This theory is valid as long as the electrode size is much larger than the Debye length $(\sim 10 \mathrm{~nm})$. Consequently, higher speeds can still be obtained by decreasing the electrode width. Nevertheless, the theory is correct for low applied voltages and we have not a robust prediction beyond this approximation

Although the normal mode of pumping can be explained qualitatively by the linear theory, the reversal of pumping which is seen in both types of devices cannot. This reversal of fluid at higher voltages and frequencies has also been observed by Studer et al [20], who studied the pumping of an asymmetric electrode device. They also found that the velocity in the reverse pumping peaked at a certain frequency for a constant voltage. This frequency was higher than observed in the present work. In our case for the traveling-wave array, both reversal and normal modes show the highest velocities at frequencies around $1 \mathrm{kHz}$. In [20] the maximum velocity for the normal pumping was measured around $1 \mathrm{kHz}$, and the maximum speed in the reverse motion was found around 50 $\mathrm{kHz}$. The transition from normal to reverse pumping was observed increasing the frequency rather than increasing the voltage, as in our case.

These flows can not be ascribed to the induction EHD pump realized by Fuhr et al in microsystems [6] because our voltages are too low to generate gradients of conductivity through Joule heating [25]. In the induction EHD pumping of dielectric liquids, forward and reverse flows have been predicted theoretically [26] for liquid/vapor medium. In spite of being very different, we can make an analogy taking into account the sign of the charges induced in the ac electroosmotic model. In this case, the mechanism could be analogous to the induction EHD pumping of dielectric liquids in the attraction mode [26]. For this mode, the theory predicts that the fluid moves in the direction of the traveling wave and no change in flow direction occurs. An instability in the flow speed is predicted for increasing frequency. However, the velocity should then be of the order of the wave phase velocity, which in our case is of the order of $160 \mathrm{~mm} / \mathrm{s}$. This phase velocity is much greater than the typical fluid velocity in our experiments (smaller than $1 \mathrm{~mm} / \mathrm{s})$.

In $[8,23]$, it was proposed that Faradaic currents cause charging of the double layer and can give rise to reverse pumping. Washabaugh et al [27] observed forward and backward pumping of semi-insulating liquids (i.e. leaky dielectrics) pumped by a traveling-wave potential. They assumed that charge injection was responsible for the fluid motion. Although, these liquids are quite different to conducting electrolytes, there might be some similarities in the origin of the flow behavior. Whether or not the reverse pumping is caused by this mechanism in both devices is an open question that needs to be solved.

\section{ACKNOWLEDGMENT}

We acknowledge the financial support of the Spanish government agency DGCyT under contract BFM2003-01739.

\section{REFERENCES}

[1] H.A. Stone, A.D. Stroock, and A. Ajdari. "Engineering flows in small devices: Microfluidics towards a lab-on-a-chip". Ann. Rev. Fluid Mech., Vol. 36, pp. 381-411, 2004.

[2] H.T.G. van Lintel, F.C.M. van de Pol and S. Bouwstra "A piezoelectric micropump based on micromachining silicon", Sensors Actuators, Vol. 15, pp. 153-167, 1988.

[3] A. Manz, C.S. Effenhauser, N. Burggraf, D.J. Harrison, K. Seiler and K. Fluri, "Electroosmotic pumping and electrophoretic separations for miniaturized chemical analysis systems", J. Micromech. Microeng., Vol. 4, pp. 257-265, 1994.

[4] G. Beni and M.A. Tenan, "Dynamics of electrowetting displays", J. Appl. Phys., Vol. 52, pp. 6011-6015, 1981.

[5] D.E. Kataoka and S.M. Troian, "Patterning liquid flow on the microscopic scale", Nature, Vol. 402, pp. 794-797, 1999.

[6] G. Fuhr, R. Hagedorn, T. Müller, W. Benecke and B. Wagner, "Microfabricated electrohydrodynamic (EHD) pumps for liquids of higher conductivity", J. Microelectro-mech. Systems, Vol. 1, pp. 141$146,1992$.

[7] A.B.D. Brown, C.G. Smith, and A.R. Rennie, "Pumping of water with AC electric fields applied to asymmetric pairs of microelectrodes". Phys. Rev. E, Vol. 63, Art. No. 016305, 2001.

[8] A. Ajdari. "Pumping liquids with asymmetric electrode arrays". Phys. Rev. E., Vol. 61, R45-R48, 2000.

[9] A. Ramos, A. González, A. Castellanos, N. G. Green, and H. Morgan. "Pumping of liquids with ac voltages applied to asymmetric pairs of microelectrodes", Phys. Rev. E., Vol. 67, Art. No. 056302, 2003.

[10] M. Mpholo, C.G. Smith and A.B.D. Brown, "Low voltage plug flow pumping using anisotropic electrode arrays", Sensors and Actuators B, Vol. 92, pp. 262-268, 2003.

[11] B. P. Cahill, L. J. Heyderman, J. Gobrecht, and A. Stemmer. "Electroosmotic streaming on application of traveling-wave electric fields", Phys. Rev. E., Vol. 70, Art. No. 036305, 2004.

[12] A. Ramos, H. Morgan, N.G. Green, A. González, A. Castellanos, "Pumping of liquids with traveling-wave electroosmosis", J. Appl. Phys., Vol. 97, Art. No. 084906, 2005.

[13] A. Ramos, H. Morgan, N.G. Green, and A. Castellanos, "AC electricfield-induced fluid flow in microelectrodes", J. Colloid Interface Sci., Vol. 217, pp. 420-422, 1999.

[14] N. G. Green, A. Ramos, A. González, H. Morgan and A. Castellanos. "Fluid flow induced by non-uniform ac electric fields in electrolytes on microelectrodes. I. Experimental measurements", Phys. Rev. E., Vol. 61, pp. 4011-4018, 2000.

[15] A. González, A. Ramos, N. G. Green, H. Morgan and A. Castellanos. "Fluid flow induced by non-uniform ac electric fields in electrolytes on microelectrodes. II. A linear double-layer analysis", Phys. Rev. E., Vol. 61, pp. 4019-4028, 2000. 
[16] N. G. Green, A. Ramos, A. González, H. Morgan, and A. Castellanos. "Fluid flow induced by nonuniform ac electric fields in electrolytes on microelectrodes. III. Observation of streamlines and numerical simulation”, Phys. Rev. E., Vol. 66, Art. No. 026305, 2002.

[17] M.Z. Bazant and T.M. Squires, "Induced-charge electrokinetic phenomena: Theory and microfluidic applications", Phys. Rev. Lett., Vol. 92, Art. No. 066101, 2004.

[18] T.M. Squires and M.Z. Bazant, "Induced-charge electro-osmosis", J. Fluid Mech., Vol. 509, pp. 217-252, 2004

[19] V.G. Levich, Physicochemical Hydrodynamics. Prentice-Hall, Englewood Cliffs, N.J., 1962.

[20] V. Studer, A. Pepin, Y. Chen, A. Ajdari. "An integrated AC electrokinetic pump in a microfluidic loop for fast and tunable flow control", The Analyst, Vol. 129, pp. 944-949, 2004.

[21] Y. Huang, X.B. Wang, J.A. Tame and R. Pethig. "Electrokinetic behaviour of colloidal particles in travelling electric fields - Studies using yeast cells", J. Phys. D: Appl. Phys., Vol. 26, pp. 1528-1535, 1993.
[22] H. Morgan, A.G. Izquierdo, D. Bakewell, N.G. Green, A. Ramos. "The dielectrophoretic and traveling wave forces generated by interdigitated electrode arrays: analytical solutions using Fourier series", J. Phys. D: Appl. Phys., Vol. 34, pp. 1553-1561, 2001.

[23] D. Lastochkin, R. Zhou, P. Wang, Y. Ben, and H. Chang "Electrokinetic micropump and micromixer design based on ac Faradaic polarization", J. Appl. Phys., Vol. 96, pp. 1730-1733, 2004.

[24] R.M. Ehrlich and J.R. Melcher, "Bipolar model for traveling-wave induced non-equilibrium double-layer streaming in insulating liquids, Phys. Fluids, Vol. 25, pp. 1785-1793, 1982.

[25] A. Castellanos, A. Ramos, A. González, N.G. Green and H. Morgan, "Electrohydrodynamics and dielectrophoresis in microsystems: scaling laws", J. Phys. D: Appl. Phys., Vol. 36, pp. 2584-2597, 2003.

[26] M.Wawzyniak and J.Seyed-Yagoobi, "Stability of electrohydrodynamic induction pumping of stratified liquid/vapor medium in the presence of an external load”, IEEE Trans. Ind. Appl., Vol 35, pp. 1211-1217, 1999.

[27] A.P. Washabaugh, M. Zahn and J.R. Melcher, "Electrohydrodynamic traveling-wave pumping of semi-insulating liquids", IEEE Trans. Electr. Insul., Vol. 24, pp. 807-834, 1989. 